\title{
Opioid conversions and patient-controlled analgesia parameters in opioid-dependent patients
}

\author{
Naveen Eipe, MD $\cdot$ John Penning, MD
}

Received: 15 June 2010/Accepted: 30 August 2010/Published online: 28 September 2010

(C) Canadian Anesthesiologists' Society 2010

\section{To the Editor,}

While a recent Continuing Professional Development article outlined the perioperative management of patients taking opioids for chronic pain, ${ }^{1}$ others have highlighted the serious consequences of opioid use in these patients. ${ }^{2}$ Patients who need opioids for pain prior to their surgery almost always require these to be continued throughout the perioperative period. "Conversion" tables have been developed to calculate the parenteral equivalent of oral opioids. ${ }^{3}$ The conventional conversion ratio between oral and intravenous routes for morphine or hydromorphone is considered to be threefold $(3: 1)^{1,3}$; however, for patients on long-term or escalating doses of opioids, multiple conversion ratios have also been advocated. ${ }^{3,4}$

Due to the uncertainty regarding the exact conversion ratio in the individual patient, we suggest that it is best to assume that the oral to intravenous ratio is high when converting extended-release oral tablets to intravenous opioids. ${ }^{4}$ On the contrary, it is safer to use a low intravenous to oral ratio when converting from intravenous opioids to oral tablets. To estimate the new dose, we recommend that two different ratios be used depending on the "direction" of conversion, i.e., 4:1 for oral to intravenous and 1:2 for intravenous to oral, as shown in the Dual Ratio Opioid Conversion Chart we have developed to manage acute pain in opioid dependent patients (Figure). These dual ratios provide a conservative estimate for a new opioid

This work should be attributed to the Department of Anesthesiology, The Ottawa Hospital, Ottawa.

N. Eipe, MD $(\bowtie) \cdot$ J. Penning, MD

Department of Anesthesiology, The Ottawa Hospital,

Ottawa, Canada

e-mail: neipe@toh.on.ca dose, and depending on the direction of conversion, they provide a $25 \%$ or $33 \%$ reduction when compared with the conventional threefold ratio.

The second challenge in opioid-dependent patients requiring patient-controlled analgesia (PCA) is to set appropriate parameters. For those who are unable to take oral opioids in the perioperative period, we add a basal infusion for half the hourly intravenous equivalent (Figure). During the stable maintenance phase for effective analgesia, patients should not require more than two PCA boluses per hour on average. This appropriate bolus-demand frequency is dependent upon an appropriately chosen bolus size. To determine the PCA bolus size in opioid-dependent patients, the opioid dose-response should be compared with that of an opioid-naive patient in whom the standard morphine PCA bolus size is $1 \mathrm{mg}$. The magnitude of the shift of the opioid dose-response curve is calculated by dividing the daily oral morphine equivalent by $100 \mathrm{mg}$ and multiplying the standard bolus size ( $1 \mathrm{mg}$ of morphine) by this factor. For example, a patient taking $400 \mathrm{mg}$ per day of oral morphine preoperatively has approximately a fourfold $(400 / 100=4)$ increase in opioid requirement and would require a $4 \mathrm{mg}$ PCA bolus dose. For such a patient, the starting PCA settings would be a $4 \mathrm{mg}$ bolus, a basal infusion rate of $2 \mathrm{mg} \cdot \mathrm{hr}^{-1}$, a lockout interval of six minutes, and an hourly limit of $32 \mathrm{mg}$. At the end of the perioperative phase, if this patient requires $100 \mathrm{mg}$ of intravenous morphine per day for adequate analgesia, we would recommend $200 \mathrm{mg}$ of oral morphine in divided doses for oral use based on the Dual Ratio Opioid Conversion Chart.

Richebé et al. ${ }^{1}$ suggested setting the continuous (basal) infusion equivalent to the total preoperative opioid use and keeping the bolus dose unchanged. Previously, Hadi et al. ${ }^{5}$ suggested setting half of the preoperative dose as the basal and increasing the bolus dose by $50 \%$. Both ${ }^{1,5}$ of these 
Figure The Ottawa Hospital Acute Pain Service Dual Ratio Opioid Conversion Chart. Footnote: This chart defines the relative equivalent opioid drug dosage that depends on the direction of conversion. The actual dose, frequency, and duration of administration should be individualized to the patient's pain requirements and clinical profile
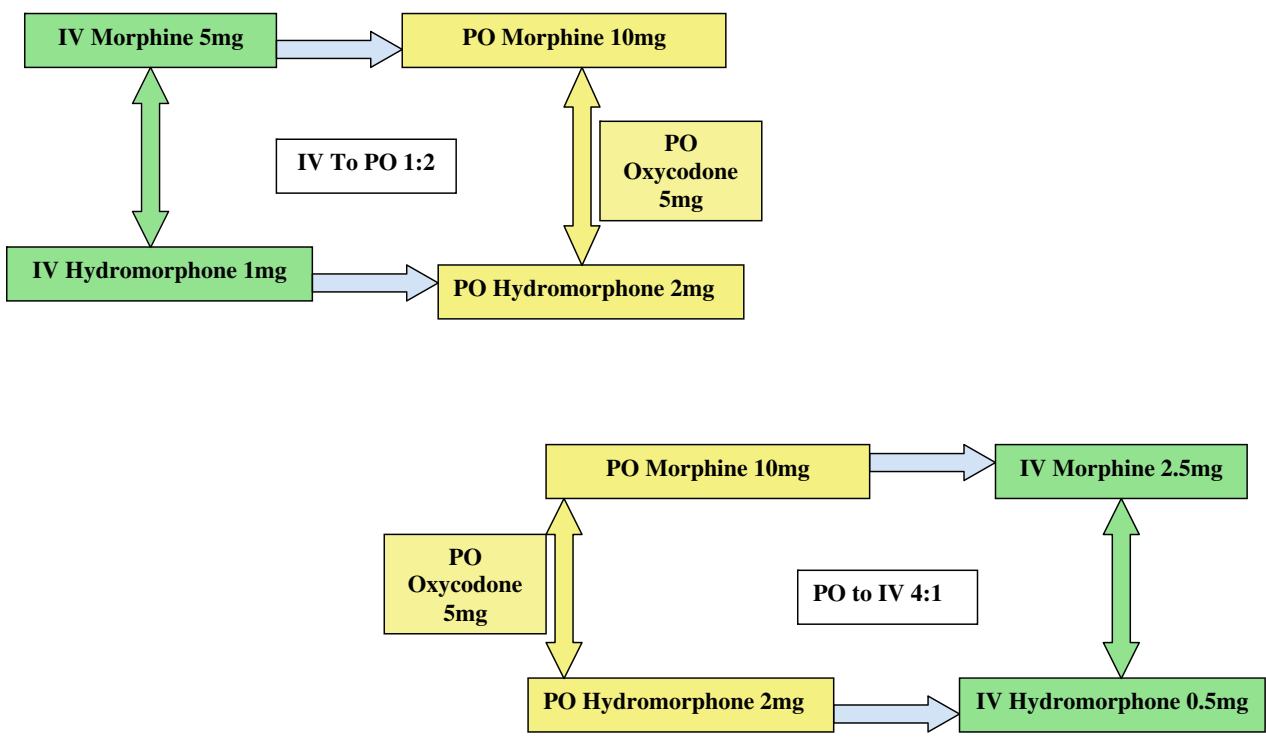

PCA settings with small bolus sizes would oblige an opioid-dependent patient to use PCA frequently to meet their hourly requirement. The small bolus sizes would provide inadequate analgesia and thus would become a cause for frustration and dissatisfaction.

With opioid-dependent patients, we recommend that perioperative requirements be determined using this Dual Ratio Opioid Conversion Chart and the PCA bolus size be adjusted using the described method of calculating the shift of the opioid response curve. While patient-to-patient variability is large and controlled studies are often difficult to perform, accumulated experience needs to be converted into evidence. We believe that our Dual Ratio Opioid Conversion Chart is a useful place to start.

Conflicts of interest and sources of support None declared.

\section{References}

1. Richebe $P$, Beaulieu P. Perioperative pain management in the patient treated with opioids: continuing professional development. Can J Anesth 2009; 56: 969-81.

2. Dhalla IA, Mamdani MM, Sivilotti ML, Kopp A, Qureshi O, Juurlink $D N$. Prescribing of opioid analgesics and related mortality before and after the introduction of long-acting oxycodone. CMAJ 2009; 181: 891-6.

3. Carroll IR, Angst MS, Clark JD. Management of perioperative pain in patients chronically consuming opioids. Reg Anesth Pain Med 2004; 29: 576-91.

4. Patanwala AE, Duby J, Waters D, Erstad BL. Opioid conversions in acute care. Ann Pharmacother 2007; 41: 255-66.

5. Hadi I, Morley-Forster PK, Dain S, Horrill K, Moulin DE. Brief review: Perioperative management of the patient with chronic noncancer pain. Can J Anesth 2006; 53: 1190-9. 